\title{
Fat and Moisture Content in Chinese Fried Bread Sticks: Assessment and Rapid Near-Infrared Spectroscopic Method Development
}

\author{
Zhuqing Xiao, ${ }^{1}$ Keqiang Lai, ${ }^{1}$ Rui Du, ${ }^{1}$ Yungang Shen, ${ }^{1}$ Xiaohua Sun, ${ }^{1}$ Yun Pan, \\ Barbara A. Rasco, ${ }^{2}$ and Yiqun Huang ${ }^{1,2}$ \\ ${ }^{1}$ College of Food Science and Technology, Shanghai Ocean University, No. 999 Hucheng Huan Road, LinGang New City, \\ Shanghai 201306, China \\ ${ }^{2}$ School of Food Science, Washington State University, Pullman, WA 99164, USA \\ Correspondence should be addressed to Yiqun Huang; yiqunh@hotmail.com
}

Received 14 March 2013; Accepted 20 May 2013

Academic Editor: Christoph Krafft

Copyright ( 2013 Zhuqing Xiao et al. This is an open access article distributed under the Creative Commons Attribution License, which permits unrestricted use, distribution, and reproduction in any medium, provided the original work is properly cited.

Fried bread sticks (FBS) are one of the most widely consumed deep fried food products in China. Understanding the fat and moisture content in FBS will help consumers make healthy food choices as well as assist food processors to provide FBS with desirable quality. Rapid Fourier transform near-infrared methods (FT-NIR) were developed for determining fat and moisture content in FBS collected from 123 different vendors in Shanghai, China. FBS samples with minimum sample preparation (either finely or coarsely ground) were used for NIR analyses. Spectra of FBS were treated with different mathematic pretreatments before being used to build models between the spectral information and fat (7.71\%-30.89\%) or moisture (17.39\%-32.65\%) content in FBS. Finely ground samples may lead to slightly more robust PLS models, but the particle sizes of ground FBS samples did not seriously affect the predictability of the models with appropriate mathematical treatments. The fat and moisture content in FBS predicted by FT-NIR methods had very good correlation with their values determined via traditional methods (fat, $R^{2}=0.965$; moisture, $R^{2}=0.983$ ), which clearly indicated that FT-NIR methods could be used as an effective tool for rapid determination of fat and moisture content in FBS.

\section{Introduction}

Chinese fried bread sticks (FBS), also known as youtiao or Chinese crullers, are very popular Chinese breakfast foods. Made from deep frying dough with leavening agents, FBS have delicious flavor and unique texture. FBS are chewy and crispy outside, but moist, light, and very porous inside. As a traditional Chinese breakfast food, each morning freshly made FBS are available from street vendors everywhere across the nation. Even fast-food giants, such as KFC, offer FBS in China to win over its local customers. Because of their desirable flavor, being easy-to-make, and of affordable price, FBS are probably the most widely consumed deep fried food in China [1].

One of the consumers' major concerns over FBS is their high fat content, a common issue associated with deep fried foods. Though fat is essential to a healthy diet and is important to provide food with desirable flavor and textural properties, but a high intake of fat may lead to an increase of health risks, such as obesity and chronic heart diseases [2]. With China's rapid economic development, Chinese consumers become increasingly aware of the health risks of high fat diets. Understanding the fat content of traditional Chinese foods like FBS will help consumers make healthy food choices. In addition, fat together with moisture content in FBS is an important factor affecting the quality of FBS. Understanding the fat and moisture contents in FBS will assist food processors to provide FBS with less fat content and desirable sensory properties.

The objective of this study was to evaluate the fat and moisture content of FBS collected from both street vendors and fast-food restaurants in China. In addition to applying traditional AOAC methods for determination of fat and 
moisture content in FBS, a Fourier transform near-infrared (FT-NIR) spectroscopic method was developed for their rapid analyses. As a rapid, nondestructive, and environmentally friendly method that does not need to use any chemicals for sample preparation, NIR has been applied in composition analysis of various food and agricultural products [3], particularly successful for moisture $[4,5]$ and fat analyses $[5,6]$ in various food systems. Although the basic ingredients for FBS were similar, mainly consisting of wheat flour, water, frying oil, and leaving agents, the ratio of these ingredients as well as the deep frying procedures (such as time, temperature) vary greatly among different street vendors and fast-food restaurants, which lead to great variation in fat and moisture in FBS. The use of NIR method to replace the traditional wet chemistry analyses for fat and moisture content in deep fried food will greatly help food processors, food researchers, and government agents for rapid screening of a large number of food samples.

\section{Materials and Methods}

2.1. Sample Collection and Preparation. Chinese fried bread sticks were collected from a total of 123 different vendors in seven districts (including Changning, Zhabei, Xuhui, Putuo, Jing'an, Yangpu, and Pudong New Districts) of Shanghai city, China. Among them, 84 samples were collected from street vendors, and 36 samples were collected from fast-food restaurants (including KFC, Yonghe King, Yonghe Soya Bean, and New Asia Snack). In addition, two samples were collected from food courts of grocery stores, and one was collected from school cafeteria. Seven pieces of FBS (about $50 \mathrm{~g}$ a piece) were purchased from each vendor.

Four pieces of FBS from each vendor (the other three were saved for other studies) were ground into two different particle sizes. A Grindomix GM 200 knife mill (Retsch Inc., Haan, Germany) was used to grind (5000 rpm, 20 seconds) FBS to particle sizes of about $500 \mu \mathrm{m}$, while a homogenizer (8010 s, Waring, Inc., Torrington, Connecticut, USA) was used (at high speed for 20 seconds) to obtain particle sizes ranging from about 1000 to $2000 \mu \mathrm{m}$. The ground FBS particles were sealed in polyethylene bags and stored at $-50^{\circ} \mathrm{C}$ before further analyses.

2.2. Determination of Fat and Moisture in FBS. A solvent extraction-gravimetric method based upon the modified AOAC Method 945.16 [7] was used to determine the fat content of FBS. The fat of finely ground FBS (about $2 \mathrm{~g}$ ) was extracted for $2.5 \mathrm{~h}$ by using a crude fat analyzer (model SZF06, Jiading Food \& Oil Instrument Ltd., Shanghai, China) with diethyl ether as solvent. Then, the solvent was evaporated at $50^{\circ} \mathrm{C}$ and the vessel containing residue was dried in oven (GZX-9146 MBE, Boxun industrial Ltd., Shanghai, China) for $1 \mathrm{~h}$ at $105^{\circ} \mathrm{C}$. The fat content of FBS was reported as the percentage of the residue in the vessel to the FBS sample weight. Duplicate analyses were conducted for each sample.

The moisture content of FBS was determined by using an oven dried method based upon the AOAC Method 945.15 [8]. In brief, about $2 \mathrm{~g}$ of finely ground FBS was dried for $3 \mathrm{~h}$ at $105^{\circ} \mathrm{C}$ in oven. The moisture content was calculated as the percentage of the sample weight loss due to the oven drying to the sample weight. Duplicate analyses were carried out for each sample.

The root mean square error of the reference (RMSR) was used to evaluate the precision of the fat and moisture content determined with the conventional methods, which was expressed as

$$
\mathrm{RMSR}=\sqrt{\frac{\sum_{i=1}^{n} \mathrm{SS}_{i}}{2 n}} .
$$

In (1), $\mathrm{SS}_{i}$ is the sum of the squares of the difference of each analysis value from the mean reference value of a tested sample- $i$, and $n$ is the number of samples $(n=123)$.

2.3. NIR Spectral Collection. A Nicolet 6700 spectrometer (Thermo Fisher Scientific Inc., Waltham, MA, USA) equipped with a NIR integrating sphere and InGaAs detector was used to obtain FT-NIR spectra (1000-2500 nm) of FBS. About $3 \mathrm{~g}$ of ground FBS was placed into the sample cup to collect spectra. Each sample was scanned 32 times at a resolution of $8 \mathrm{~cm}^{-1}$. The background was also scanned 32 times at a resolution of $8 \mathrm{~cm}^{-1}$ and deducted from each sample spectrum automatically. After each measurement, the sample cup was cleaned with isopropanol. Spectra were acquired for both coarsely and finely ground FBS particles.

2.4. Data Analysis. Data analyses were conducted by using Delight 3.2 software package (DSquared Development Inc., LaGrande, OR, USA). Spectral data were preprocessed to reduce or eliminate the interference of noise and other irrelevant background information, as well as to help separate overlapped spectral information often associated with NIR spectra. Different algorithms including binning, smoothing, derivative transformation (1st and 2nd), polynomial subtraction (1st and 2nd), multiplicative scatter correction (MSC), and standard normal variate (SNV) were employed, and the results were compared to choose the best spectral preprocessed method [9-12]. The final spectral preprocessing algorithm used for fat analysis was smoothing and then SNV, while for moisture analysis was smoothing and then first polynomial subtraction (FPS).

Partial least squares (PLS) regression was applied to correlate spectral data with fat or moisture content in FBS $[13,14]$. All samples $(n=123)$ were selectively divided into calibration $(n=82)$ and prediction $(n=41)$ data sets. Samples were ranked in the order of increasing fat or moisture content, and then every second sample was selected for the prediction set, and the rest of samples were used for the model calibration. During the model calibration, leaveone-out cross-validation was used to help decide the number of latent variables used for the final model. The model was then used to predict the fat or moisture content for the samples in the prediction set base upon their spectral data. The performance of the PLS model was evaluated by using coefficient of determination $\left(R^{2}\right)$, standard error of prediction (SEP), and the RPD (the ratio of the standard deviation to the SEP) $[15,16]$. 


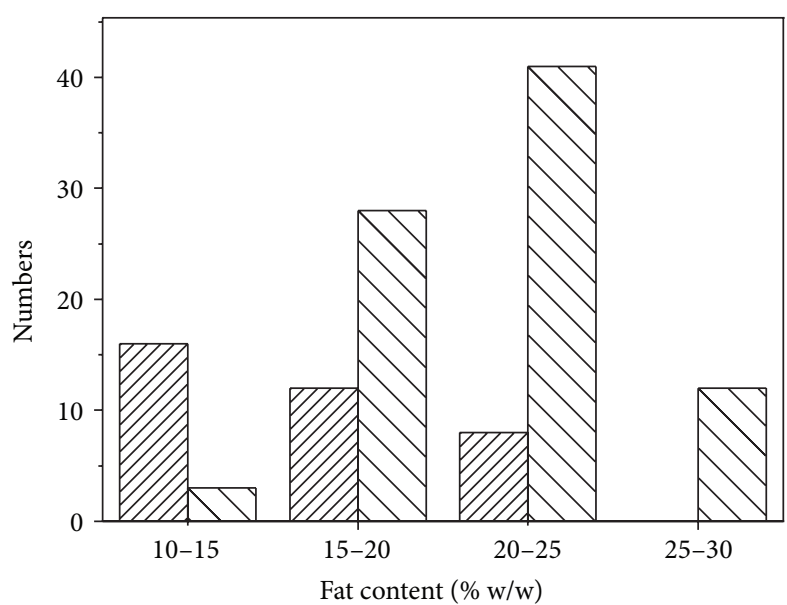

Z7] Restaurant $\triangle \nabla$ Street vendors

(a)

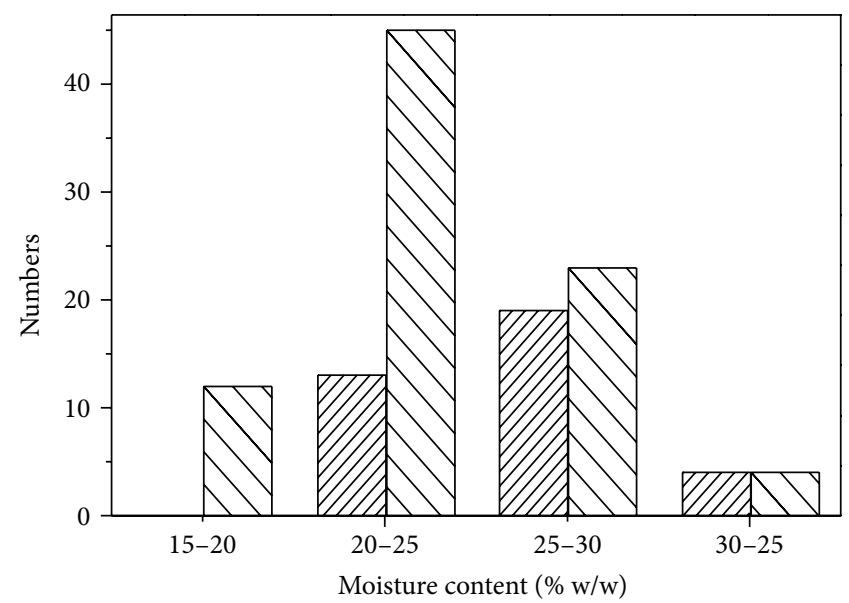

ZZJ Restaurant $\triangle \nabla$ Street vendors

(b)

Figure 1: The distribution of (a) fat and (b) moisture content in FBS collected from fast-food restaurants and street vendors.

TABLE 1: Fat content of FBS collected from different vendors.

\begin{tabular}{lcccccc}
\hline & Sample size & $\operatorname{Min}^{\mathrm{a}}$ & $\operatorname{Max}^{\mathrm{a}}$ & $\operatorname{Mean}^{\mathrm{a}}$ & $\operatorname{Median}^{\mathrm{a}}$ & $\mathrm{Std}^{\mathrm{a}, \mathrm{b}}$ \\
\hline Restaurants & 36 & 11.04 & 24.13 & 16.67 & 15.86 & 3.92 \\
Street vendors & 84 & 13.48 & 30.89 & 21.32 & 21.53 & 3.79 \\
Others & 3 & 7.71 & 10.75 & 9.26 & 9.33 & 1.52 \\
\hline Total & 123 & 7.71 & 30.89 & 19.67 & 19.75 & 4.63 \\
\hline
\end{tabular}

${ }^{\mathrm{a}}$ The units were $\%(\mathrm{w} / \mathrm{w})$.

${ }^{\mathrm{b}}$ Standard deviation of samples.

\section{Results and Discussion}

3.1. Fat and Moisture Content in FBS. The root mean square error of the reference was $0.21 \%$ for fat and $0.18 \%$ for water. As shown in Table 1, there was great disparity in the fat content of FBS collected in Shanghai city. The overall fat content of FBS ranged from $7.71 \%$ to $30.89 \%$, and the mean and median of fat content were $19.67 \%$ and $19.75 \%$, respectively. The mean and median of fat content for FBS from street vendors were $27.89 \%$ and $35.75 \%$ higher than those collected from the fastfood restaurants. About 44\% (16 out of 36) of FBS samples collected from fast-food restaurants contained less than $15 \%$ of fat, and about $22 \%$ ( 8 out of 36 ) had more than $20 \%$ of fat; however, only $3.6 \%$ (3 out of 84 ) of FBS from street vendors contained less than $15 \%$ of fat, and $63 \%$ of FBS (53 out of 84) from street vendors contained more than $20 \%$ of fat (Figure 1(a)).

Table 2 shows the moisture content in FBS from the fastfood restaurants (20.97-31.08\%) and from the street vendors (17.39-31.51\%). The mean and median of moisture content in FBS from the fast-food restaurants were $26.44 \%$ and $26.39 \%$, respectively, about $11 \%$ higher than those from the street vendors (mean, 23.85\%; median, 23.82\%). Furthermore, about $68 \%$ (57 out 84 samples) of FBS from street vendors contained less than $25 \%$ water, but $64 \%$ (23 out of 36 samples) of FBS
TABLE 2: Moisture content of FBS collected from different vendors.

\begin{tabular}{lcccccc}
\hline & Sample size & $\operatorname{Min}^{\mathrm{a}}$ & $\operatorname{Max}^{\mathrm{a}}$ & $\operatorname{Mean}^{\mathrm{a}}$ & $\operatorname{Median}^{\mathrm{a}}$ & $\mathrm{Std}^{\mathrm{a}, \mathrm{b}}$ \\
\hline Restaurants & 36 & 20.97 & 31.08 & 26.44 & 26.39 & 2.55 \\
Street vendors & 84 & 17.39 & 31.51 & 23.85 & 23.82 & 3.33 \\
Others & 3 & 31.87 & 32.65 & 32.21 & 32.10 & 0.42 \\
\hline Total & 123 & 17.39 & 32.65 & 24.81 & 24.53 & 3.49
\end{tabular}

${ }^{a}$ The units were $\%(\mathrm{w} / \mathrm{w})$.

${ }^{\mathrm{b}}$ Standard deviation of samples.

from fast-food restaurants contained higher than $25 \%$ water (Figure 1(b)).

Only three FBS samples were collected from food courts or school cafeteria other than fast-food restaurants or street vendors since it is rare for food courts in grocery stores or malls to provide breakfast food like FBS (Tables 1 and 2). These three samples contained the lowest fat (7.71$10.75 \%)$ and highest moisture content (31.87-32.65\%), and further investigation is needed to understand the sources and eventually the processing methods of these FBS samples.

The fat and moisture content in FBS had no strict linear relationship $\left(R^{2}=0.452\right)$, but the fat content tended to increase as the moisture content decreased (Figure 2). During the deep frying process, high temperature caused evaporation of some water and left porous structure in the food, which allowed oil to migrate inside the food. Generally, the more the water is lost, the more the oil is being absorbed. Therefore, the fat and moisture content in FBS had somewhat inverse linear relationship.

3.2. Spectral Feature of FBS. Figure 3 shows the NIR spectra of FBS and its main ingredients including wheat flour, water, and frying oil (soy oil was used for illustration here). Basically, the spectral feature of FBS reflected the chemical compositions of its three major ingredients. Since wheat flour 


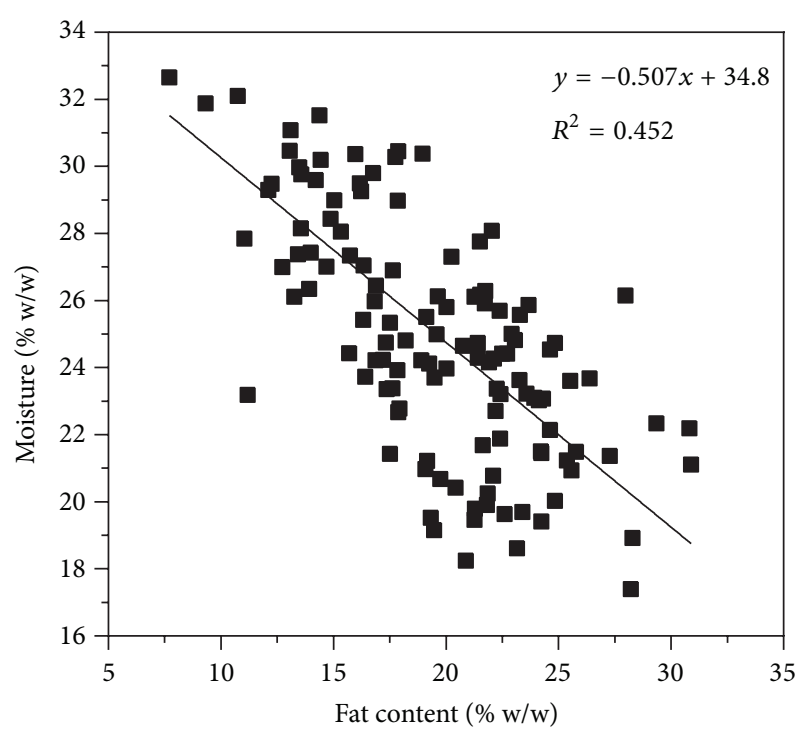

FIGURE 2: Plots of moisture content versus fat content of FBS.

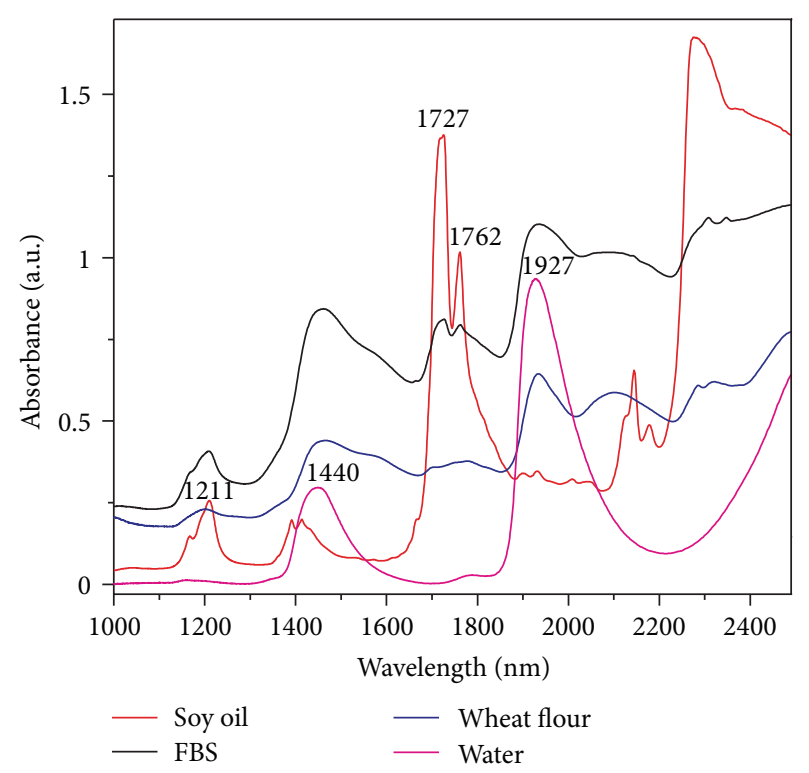

FIGURE 3: FT-NIR spectra of FBS and their main ingredients including wheat flour, water, and soy oil.

(normally contains about $75 \%$ of carbohydrate and $13 \%$ of water) is the major ingredient for FBS, the shape of the NIR spectra of FBS and wheat flour were similar, except that the spectra of FBS contained some extra bands due to oils absorbed during frying process. The peaks at around 1727 , $1762 \mathrm{~nm}$ in the spectrum of FBS were assigned to the first overtone of methyl and methylene $\mathrm{C}-\mathrm{H}$ stretching vibration for oils, and their second overtone was observed at around $1211 \mathrm{~nm}[17,18]$. The absorbance at around $1440 \mathrm{~nm}$ and $1927 \mathrm{~nm}$ in the spectrum of FBS could be due to the vibrations of the $\mathrm{O}-\mathrm{H}$ groups in carbohydrates and water, since FBS contained both carbohydrates and water and these two components provide similar $\mathrm{O}-\mathrm{H}$ stretching and bending modes at around $1440 \mathrm{~nm}$ and $1927 \mathrm{~nm}$ [19-22].

Figure 4 shows some representative FT-NIR spectra of FBS with different fat content as well as their spectra after SNV transformations. Although it is hard to discern the difference among three original spectra of FBS because of the baseline shift (Figure 4(a)), it was quite obvious that the absorbance intensity of three major triglyceride bands at 1211, 1727, and $1762 \mathrm{~nm}$ increased as the fat content increased based upon their SNV treated spectra (Figure 4(b)). This clearly showed the advantages of applying spectral pretransformation, such as SNV, for analysis of fat in FBS.

Table 3 shows the linear relationship between fat content in FBS and the absorbance intensity of FBS at the wavelengths corresponding to the three major triglyceride bands. Again, after SNV treatment, the $R^{2}$ values between the fat content and absorbance intensity at three major triglyceride peaks were greatly improved. For examples, the $R^{2}$ of fat content and absorbance intensity at $1211 \mathrm{~nm}$ were improved from $0.339-0.472$ to $0.836-0.842$ and for that at $1727 \mathrm{~nm}$ were improved from $0.436-0.526$ to $0.701-0.731$. For finely ground FBS samples, the $R^{2}$ values were slightly higher than that for the coarsely ground FBS samples.

The moisture content in FBS had no obvious relationship with the absorbance intensity of $\mathrm{O}-\mathrm{H}$ vibrations at around 1440 and $1927 \mathrm{~nm}$. For example, for the three FBS samples used in the Figure 4(b), their moisture content $(19.51 \%$ $32.65 \%)$ was inversely related with their fat content $(7.71 \%-$ $30.89 \%$ ), but the difference in moisture content among these three samples was not reflected on the absorbance intensity due to $\mathrm{O}-\mathrm{H}$ vibrations in their NIR spectra. This is mainly because of the overlapping of $\mathrm{O}-\mathrm{H}$ vibrations of carbohydrate and water molecules.

3.3. FT-NIR Models for Fat and Moisture Content. As shown in Table 4, the PLS models with the use of four or five latent variables exhibited good predictability for fat content in FBS $\left(R^{2}=0.960-0.965\right.$, SEP $=0.93-0.96 \%$, and RPD = 4.89-5.04), and the use of finely ground FBS yielded similar results as the coarsely ground FBS for fat determination. For moisture analysis, the use of finely ground FBS resulted in a somewhat better PLS model $\left(R^{2}=0.983\right.$, SEP $=0.46$, RPD $=7.64, \mathrm{LV}=6)$ than that of using coarsely ground sample $\left(R^{2}=0.977\right.$, SEP $=0.56, \mathrm{RPD}=6.29$, and LV $\left.=7\right)$, but PLS models based on both finely ground and coarsely ground FBS samples resulted in RPD values higher than 6, which clearly showed that FT-NIR method could be used to accurately predict moisture content in FBS [15]. Figure 5 shows the fat or moisture content in FBS predicted by the best PLS models against their reference value (fat, $R^{2}=0.965$; moisture, $R^{2}=$ 0.983), which again indicated that NIR methods could be used as an effective tool for rapid determination of fat and moisture content in FBS.

\section{Conclusions}

The fat content $(7.71 \%-30.89 \%)$ of FBS collected from Shanghai city varied greatly, and the mean and median of fat 


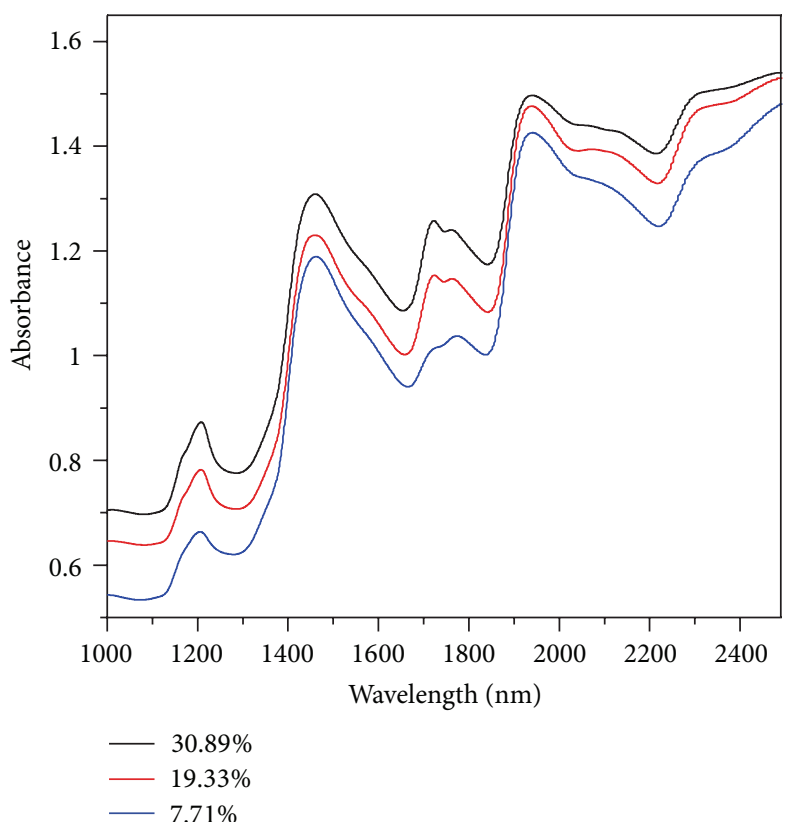

(a)

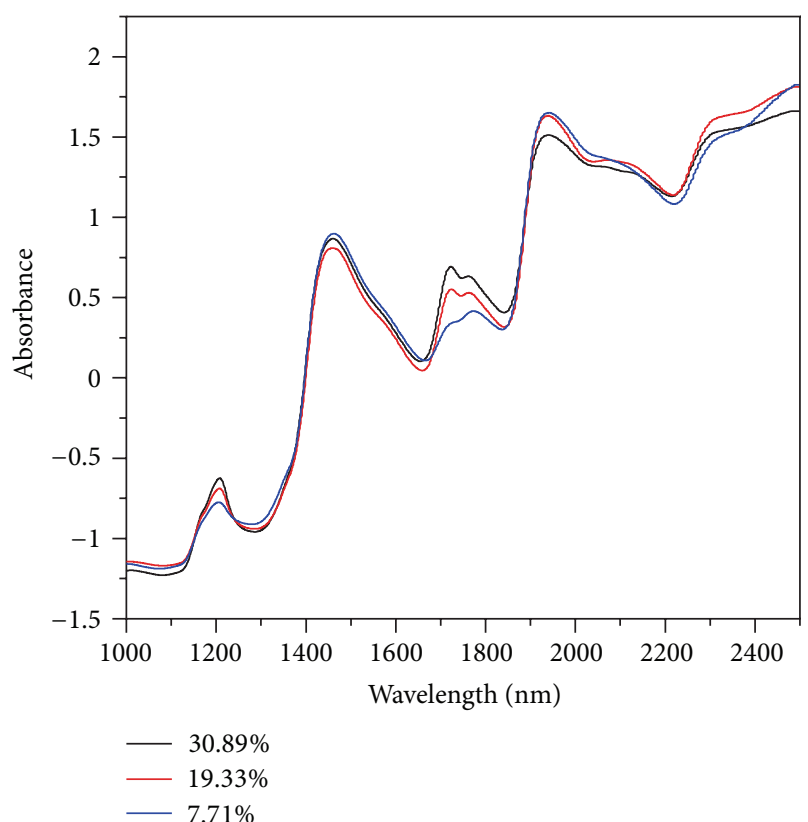

(b)

FIGURE 4: (a) Representative spectra of FBS with different fat contents and (b) their spectra after being treated with SNV.

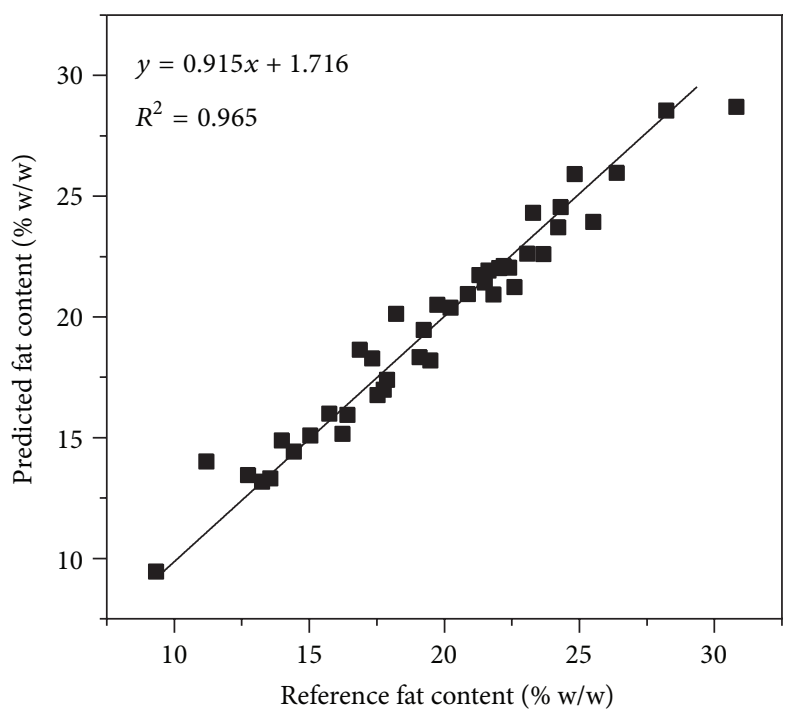

(a)

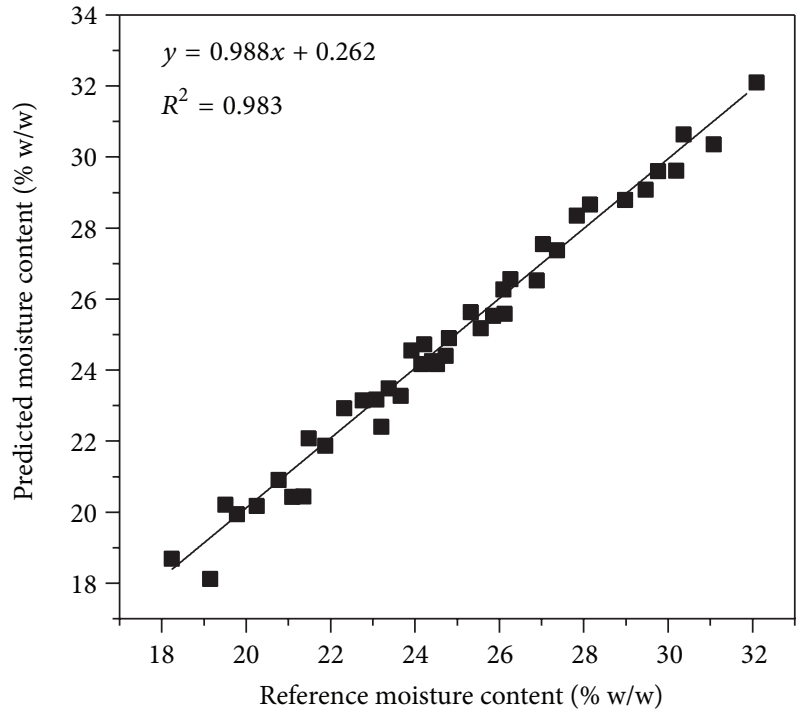

(b)

FIGURE 5: Plots of (a) fat and (b) moisture content predicted with PLS models versus their values determined with the standard method.

content for FBS from street vendors were 27.89\% and 35.75\% higher than those collected from the fast-food restaurants. In addition, the fat and moisture content (17.39\%-32.65\%) in FBS had somewhat inverse linear relationship. The higher the fat content, the lower the moisture content in FBS. The mean and median of moisture content in FBS from the fastfood restaurants were $26.44 \%$ and $26.39 \%$, respectively, about $11 \%$ higher than those from the street vendors (mean, 23.85\%; median, 23.82\%).
The use of FT-NIR methods for analyses of FBS collected from 123 different vendors in Shanghai city clearly showed that FT-NIR could be a very effective tool for determination of fat and moisture content in FBS with minimum sample preparation. Finely ground samples may lead to slightly more robust PLS models, but the particle sizes of ground FBS samples did not seriously affect the predictability of the models with appropriate mathematical treatments, such as SNV and FPS [23]. 
TABLE 3: Linear relationship between fat content and absorbance intensity at three major peaks of triglycerides for original and mathematical transformed spectra of both finely and coarsely ground FBS sample.

\begin{tabular}{lcccc}
\hline & & \multicolumn{2}{c}{ Wavelength (nm) } \\
& & 1211 & 1727 & 1762 \\
\hline Original spectra & $\mathrm{LE}^{\mathrm{a}}$ & $y=0.50 x-0.18^{\mathrm{b}}$ & $y=0.49 x-0.36$ & 0.526 \\
(finely ground) & $R^{2}$ & 0.472 & $y=0.50 x-0.04$ & 0.447 \\
SNV treated spectra & $\mathrm{LE}$ & $y=1.56 x+1.31$ & 0.731 & $y=0.57 x-0.08$ \\
(finely ground) & $R^{2}$ & 0.842 & $y=0.47 x-0.34$ & 0.576 \\
Original spectra & $\mathrm{LE}$ & $y=0.40 x-0.11$ & 0.436 & $y=0.45 x-0.31$ \\
(coarsely ground) & $R^{2}$ & 0.339 & $y=0.49 x-0.04$ & 0.357 \\
SNV treated spectra & $\mathrm{LE}$ & $y=1.52 x+1.28$ & 0.701 & $y=0.55 x-0.07$ \\
(coarsely ground) & $R^{2}$ & 0.836 & & 0.537 \\
\hline
\end{tabular}

${ }^{\mathrm{a}} \mathrm{LE}$ was linear equation;

${ }^{\mathrm{b}} x$ was the absorbance intensity at a specific wavelength, and $y$ was fat content in FBS.

TABLE 4: Summary of predictability of PLS models for fat and moisture in finely or coarsely ground FBS.

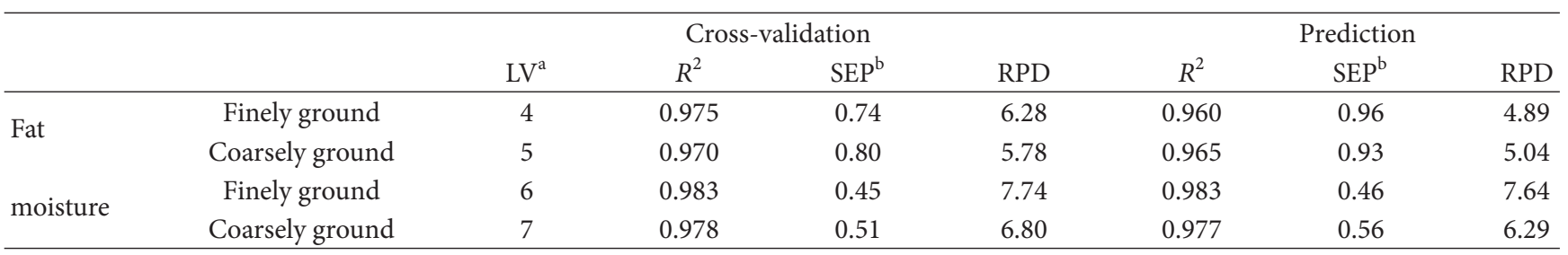

${ }^{a}$ Number of latent variables (LV).

${ }^{\mathrm{b}}$ The unit was $\%(\mathrm{w} / \mathrm{w})$.

\section{Acknowledgments}

This research was supported by the Science and Technology Commission of Shanghai Municipality (Project 09PJ1405200 and 09320503800). Additional support for this research was provided by the Leading Academic Discipline Project of Shanghai Municipal Education Commission (Project J50704) and Shanghai Ocean University (A-2400-09-0145).

\section{References}

[1] Y. Zhang and Y. Zhang, "Study on reduction of acrylamide in fried bread sticks by addition of antioxidant of bamboo leaves and extract of green tea," Asia Pacific Journal of Clinical Nutrition, vol. 16, no. 1, pp. 131-136, 2007.

[2] W. V. Brown, K. Fujioka, P. W. F. Wilson, and K. A. Woodworth, "Obesity: why be concerned?" American Journal of Medicine, vol. 122, no. 4, pp. S4-S11, 2009.

[3] W. F. McClure, "204 Years of near infrared technology: 18002003," Journal of Near Infrared Spectroscopy, vol. 11, no. 6, pp. 487-518, 2003.

[4] W. R. Hruschka and K. H. Norris, "Least-squares curve fitting of near infrared spectra predicts protein and moisture content of ground wheat," Applied Spectroscopy, vol. 36, pp. 261-265, 1982.

[5] M. M. Pierce and R. L. Wehling, "Comparison of sample handling and data treatment methods for determining moisture and fat in cheddar cheese by near-infrared spectroscopy," Journal of Agricultural and Food Chemistry, vol. 42, no. 12, pp. 2830-2835, 1994.

[6] R. Du, K. Lai, Z. Xiao, Y. Shen, X. Wang, and Y. Huang, "Evaluation of the quality of deep frying oils with Fourier Transform near-infrared and mid-infrared spectroscop," Journal of Food Science, vol. 77, no. 2, pp. C261-C266, 2012.

[7] AOAC Method 945.16, "Oil in cereal adjuncts: petroleum ether extraction method," in Official Methods of Analysis of AOAC International, Association of Official Analytical Chemists, Arlington, Va, USA, 17th edition, 2000.

[8] AOAC Method 945.15, "Moisture in cereal adjuncts: air oven method," in Official Methods of Analysis of AOAC International, Association of Official Analytical Chemists, Arlington, Va, USA, 17th edition, 2000.

[9] G. E. Collins, "Calculation of multivariate polynomial resultants," Journal of the Association for Computing Machinery, vol. 18 , no. 4, pp. 515-532, 1971.

[10] T. Isaksson and T. Naes, "Effect of multiplicative scatter correction (MSC) and linearity improvement in NIR spectroscopy," Applied Spectroscopy, vol. 42, no. 7, pp. 1273-1284, 1988.

[11] R. J. Barnes, M. S. Dhanoa, and S. J. Lister, "Standard normal variate transformation and de-trending of near-infrared diffuse reflectance spectra," Applied Spectroscopy, vol. 43, no. 5, pp. 772$777,1989$.

[12] H. Cen and Y. He, "Theory and application of near infrared reflectance spectroscopy in determination of food quality," Trends in Food Science and Technology, vol. 18, no. 2, pp. 72-83, 2007.

[13] D. M. Haaland and E. V. Thomas, "Partial least-squares methods for spectral analyses. 1. Relation to other quantitative calibration methods and the extraction of qualitative information," Analytical Chemistry, vol. 60, no. 11, pp. 1193-1202, 1988.

[14] H. Büning-Pfaue and S. Kehraus, "Application of near infrared spectroscopy (NIRS) in the analysis of frying fats," European Journal of Lipid Science and Technology, vol. 103, no. 12, pp. 793797, 2001. 
[15] Y. Huang, T. M. Rogers, M. A. Wenz et al., "Detection of sodium chloride in cured salmon roe by SW-NIR spectroscopy," Journal of Agricultural and Food Chemistry, vol. 49, no. 9, pp. 4161-4167, 2001.

[16] M. Lin, A. G. Cavinato, Y. Huang, and B. A. Rasco, "Predicting sodium chloride content in commercial king (Oncorhynchus tshawytscha) and chum (O. keta) hot smoked salmon fillet portions by short-wavelength near-infrared (SW-NIR) spectroscopy," Food Research International, vol. 36, no. 8, pp. 761766, 2003.

[17] R. T. Holman and P. R. Edmondson, "Near-infrared spectra of fatty acids and some related substances," Analytical Chemistry, vol. 28, no. 10, pp. 1533-1538, 1956.

[18] S. E. Kays, W. R. Windham, and F. E. Barton II, "Prediction of total dietary fiber by near-infrared reflectance spectroscopy in high-fat- and high-sugar-containing cereal products," Journal of Agricultural and Food Chemistry, vol. 46, no. 3, pp. 854-861, 1998.

[19] D. Baker, "The determination of fiber in processed cereal foods by near-infrared reflectance spectroscopy," Cereal Chemistry, vol. 60 , pp. 217-219, 1983.

[20] F. O. Libnau, O. M. Kvalheim, A. A. Christy, and J. Toft, "Spectra of water in the near- and mid-infrared region," Vibrational Spectroscopy, vol. 7, no. 3, pp. 243-254, 1994.

[21] L. E. Rodriguez-Saona, F. S. Fry, and E. M. Calvey, "Use of fourier transform near-infrared reflectance spectroscopy for rapid quantification of castor bean meal in a selection of flourbased products," Journal of Agricultural and Food Chemistry, vol. 48, no. 11, pp. 5169-5177, 2000.

[22] C. Shiroma and L. Rodriguez-Saona, "Application of NIR and MIR spectroscopy in quality control of potato chips," Journal of Food Composition and Analysis, vol. 22, no. 6, pp. 596-605, 2009.

[23] Å. Rinnan, F. V. D. Berg, and S. B. Engelsen, "Review of the most common pre-processing techniques for near-infrared spectra," Trends in Analytical Chemistry, vol. 28, no. 10, pp. 1201-1222, 2009. 

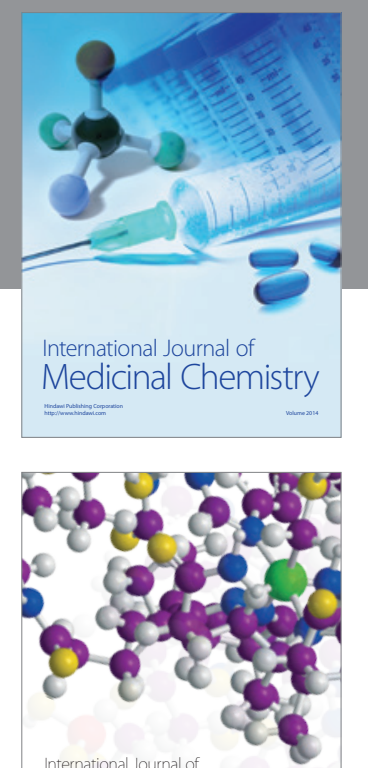

\section{Carbohydrate} Chemistry

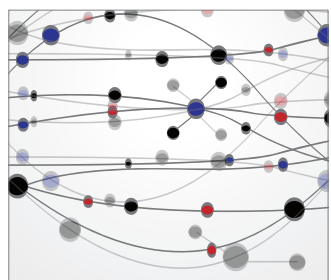

The Scientific World Journal
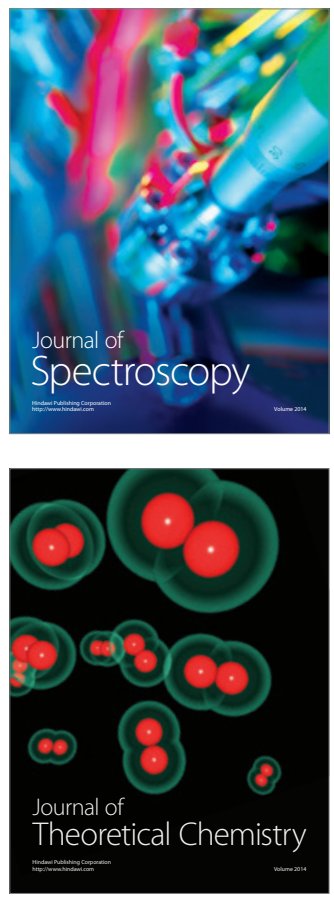
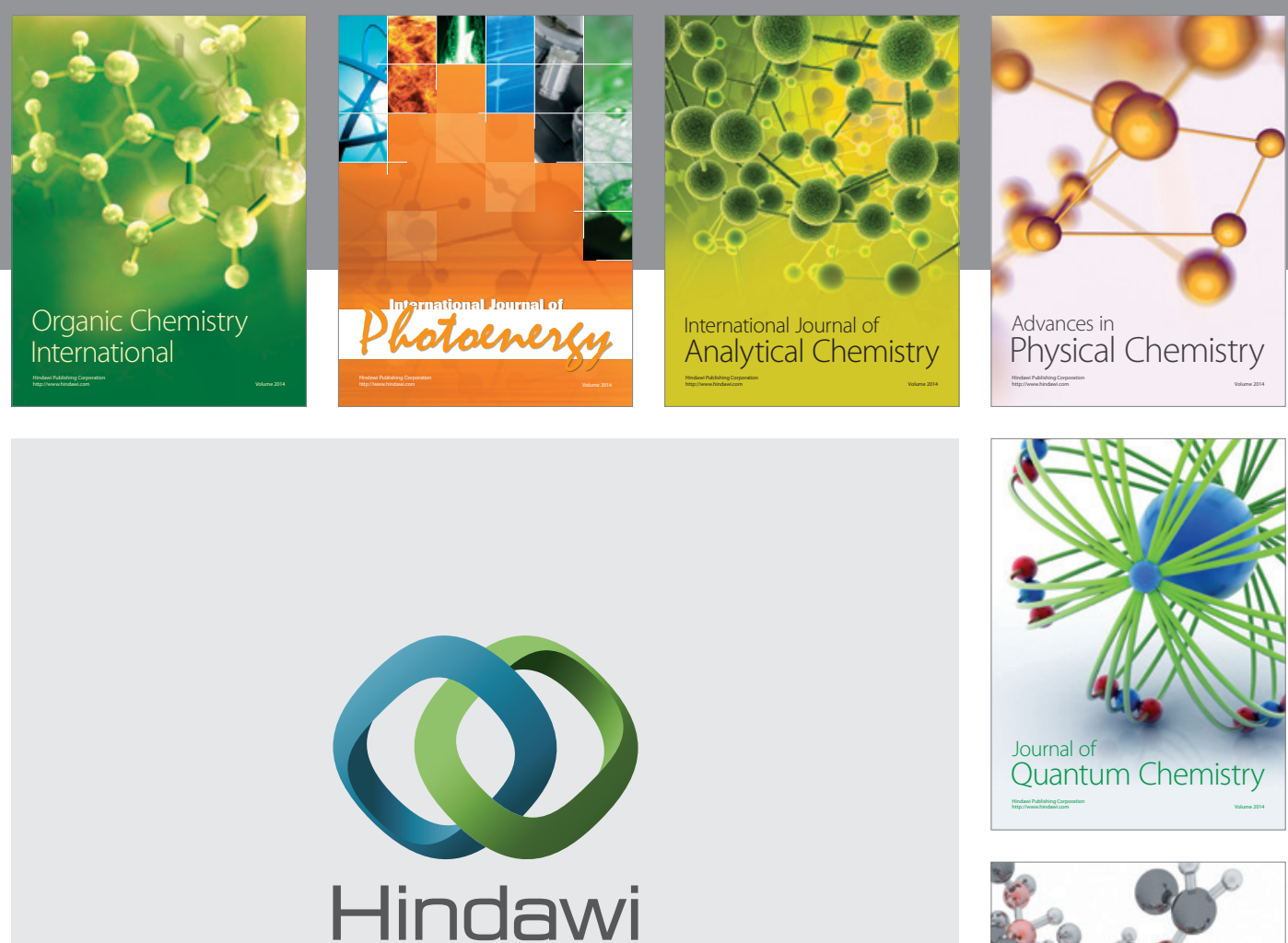

Submit your manuscripts at

http://www.hindawi.com

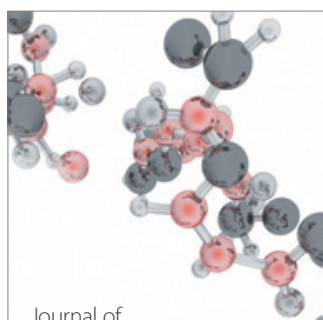

Analytical Methods

in Chemistry

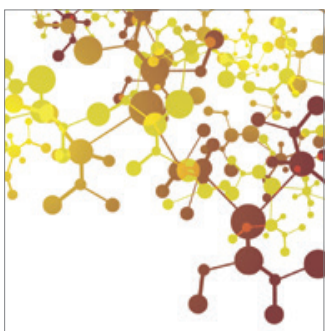

Journal of

Applied Chemistry

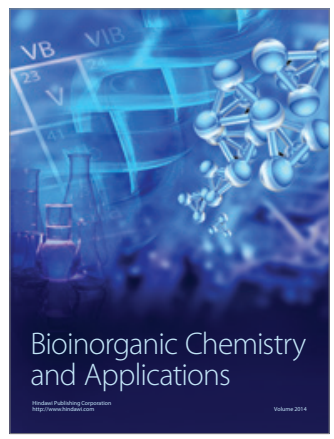

Inorganic Chemistry
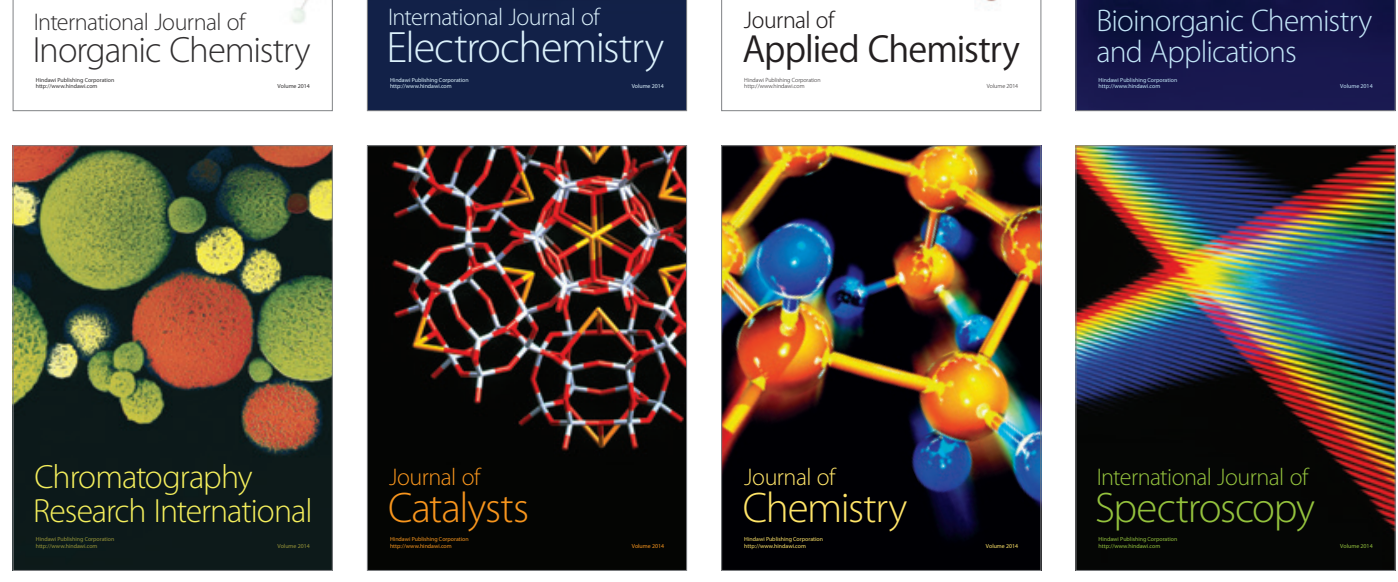